\title{
Development of a Three-Dimensional Magnifying Visual Information Display System to Support the Daily Activities of the Visually Impaired
}

\author{
Toshiaki Tanaka, Norio Kato, Tomoya Miyasaka, Masatsugu Sakajiri
}

\begin{abstract}
There are 310,000 visually impaired people in Japan, about $80 \%$ of whom are said to have low vision. Optical aids such as magnifying glasses (loupes), monocular devices, and reading magnification systems are used in daily life. However, these assistive devices are not used widely or for many purposes. Moreover, two-dimensional visual magnification also interferes depth perception and interaction in three-dimensional (3D) space. To eliminate the impediments of low vision, better design and the provision of assistive devices in a range of settings are needed. For this research, we developed and tested wearable 3D magnifying glasses designed to compensate for vision loss and support activities of daily living in older people and those with low vision. These glasses consist of a head-mounted display (HMD) with built-in miniature cameras and are capable of performing rapid 3D magnification of objects captured by the cameras before presenting these images to the display. An HMD with built-in miniature charge-coupled device cameras has a wired connection to a control terminal and is operated by a Bluetooth remote control. The operator can also operate the control terminal from an operator terminal connected by Wi-Fi. The cameras feature two horizontally installed miniature CMOS color image sensors. The display unit has a resolution at least as good as VGA (default resolution $640 \times 480$ ) at both left and right. Adjusting screws are installed at both sides to allow focus adjustment and binocular parallax. Built-in speakers were also added to enable the use of an audio guide. It was also possible to achieve specifications as follows; image processing function modes and external input display mode. In order to evaluate the effect of the specifications by using the HMD, the Questionnaire survey of the usability of the prototype HMD was conducted in $\mathbf{1 0}$ participants (9 men, 1 woman; age range, 20-22 years) with visual impairment (low vision). The results showed that the 3D-HMD might be effective for the visually handicapped person and the elderly to assist the reduced visual acuity. It was suggested that the improvement in converted visual acuity using the HMD could enhance the accuracy (e.g., precision, speed) of communication aimed at the visually impaired compared with existing devices. In the future, this HMD needs to be studied in a larger sample using several reliable test methods that take less time and thus place less burden on participants.
\end{abstract}

Index Terms - Low Vision, 3D-HMD, Communication Aid.

Toshiaki Tanaka, Institute of Gerontology, The University of Tokyo, Tokyo, Japan, Department of Physical Therapy, Faculty of Health Sciences, Hokkaido University of Science, Sapporo, Hokkaido, Japan

Norio Kato, Department of Physical Therapy, Faculty of Health Sciences, Hokkaido University of Science, Sapporo, Hokkaido, Japan

Tomoya Miyasaka, Department of Physical Therapy, Faculty of Health Sciences, Hokkaido University of Science, Sapporo, Hokkaido, Japan

Masatsugu Sakajiri, Department of Computer Science, Faculty of Health Sciences, National University Corporation Tsukuba University of Technology, Ibaraki, Japan.

\section{INTRODUCTION}

There are 310,000 visually impaired people in Japan (source: Ministry of Health, Labour and Welfare), about $80 \%$ of whom are said to have low vision. Low vision is defined as a condition in which vision loss interferes with daily life and cannot be sufficiently corrected by glasses or contact lenses. The World Health Organization defines low vision as a condition in which visual acuity is equal to or better than 0.05 and less than 0.3 , even when using corrective lenses. This causes difficulties in daily activities, in the workplace, and in other settings. Low vision is a distinct category from total blindness, and in medical terms, is a form of visual impairment. Based on figures published by the Japan Ophthalmologists Association, about 190,000 people in Japan are certified as visually impaired from grade 2 to grade 6 , under the narrowest definition, and 1,449,000 under the broadest definition. Night blindness, visual field constriction, central scotoma, photophobia, double vision, nystagmus, color blindness, ptosis, and day blindness also fall under the basic category of visual impairment. Optical aids such as magnifying glasses (loupes), monocular devices, and reading magnification systems are used in daily life. Reading magnification systems help people with low vision to read, normally by using a camera with a zoom function to display magnified images on a monitor. The basic setup consists of a monitor, controller, camera, and X-Y table on which the reading material is placed, and the most common type of reading magnification system has these elements vertically aligned. However, these assistive devices are not used widely or for many purposes. Public library services aimed at the visually impaired, and braille libraries in particular, mostly provide audio books or books translated into braille; very few libraries offer services for creating large-print copies. Furthermore, given the labor and print costs for their creation, it is not yet possible to meet the needs of the visually impaired for large-print textbooks. Two-dimensional visual magnification also interferes depth perception and interaction in three-dimensional (3D) space. To eliminate the impediments of low vision, better design and the provision of assistive devices in a range of settings are needed. The developmental study of the HMD with virtual technology for people with low vision is increasing gradually [1],[2].

For this research, we developed and tested wearable 3D magnifying glasses designed to compensate for vision loss and support activities of daily living in older people and those 
Development of a Three-Dimensional Magnifying Visual Information Display System to Support the Daily Activities of the Visually Impaired

with low vision. These glasses consist of a head-mounted display (HMD) with built-in miniature cameras and are capable of performing rapid $3 \mathrm{D}$ magnification of objects captured by the cameras before presenting these images to the display.

\section{METHODS}

The HMD system was designed and manufactured according to specifications (1) to (4) below.

(1): The HMD has built-in miniature cameras featuring miniature complementary metal-oxide semiconductor (CMOS) color image sensors (external dimensions $25 \mathrm{~mm} \mathrm{x}$ $25 \mathrm{~mm}$, horizontal angle of view 30-120 deg) and can achieve $8 \mathrm{x}$ to $10 \mathrm{x}$ magnification using digital and optical zoom. The image resolution used is at least as high as SVGA (Super Video Graphics Array).

(2): To add a white-on-black mode, functional equivalence to the standard black-on-white mode is achieved by modification of the image board and the use of control software. The following technologies of the software previously developed by Tanaka was also incorporated into the HMD [3], [4]. Eye movement is encouraged by displaying a stationary arrow (flashing) to prompt attention at the site of visual field defect or by displaying an arrow that can move right, left, up, and down.

(3): To achieve a lightweight HMD of 100-150 g, an existing HMD model was adapted for the housing and miniature cameras were used. The image processing box size was also given a compact form so that it could be handheld or worn on a waist belt.

(4): To present visual information wirelessly, wireless data transmission (Bluetooth, $2.4 \mathrm{G}$ ) is used so that visual information data can be collected and sent to the HMD.

The HMD was created using these four specifications. After its manufacture, we tested its effectiveness by conducting a monitor-based study in 10 visually-impaired individuals (9 men, 1 woman; age range, 20-22 years). Information on the participants' visual impairment status (e.g., visual acuity, visual field) was obtained before the study began.

The device was evaluated by comparing participants' ability to recognize written characters with the naked eye or using the HMD when a text prepared by the experimenter was gradually magnified from $1 \mathrm{x}$ to $10 \mathrm{x}$. The study also tested the white-on-black mode, which is considered easier to read and see by people with low vision. It is now recognized that reading acuity must also be assessed directly to ascertain the degree of difficulty of low vision reading for an individual [5]-[8]. An effective tool for doing this is the set of MNREAD Acuity charts developed by the Laboratory for Low Vision Research, University of Minnesota [9]-[11]. We therefore investigated the range of print sizes that could be recognized with the HMD using the Japanese version of the MNREAD charts (MNREAD-J) [12].

This study received the approval of the Ethics Committee of the University of Tokyo (15-249).

\section{RESULTS AND ANALYSIS}

\section{III-1. HMD manufacture}

III-1-1. System configuration (Fig.1,Fig.2)

An HMD with built-in miniature charge-coupled device cameras (Fig. 1) has a wired connection to a control terminal and is operated by a Bluetooth remote control. The operator can also operate the control terminal from an operator

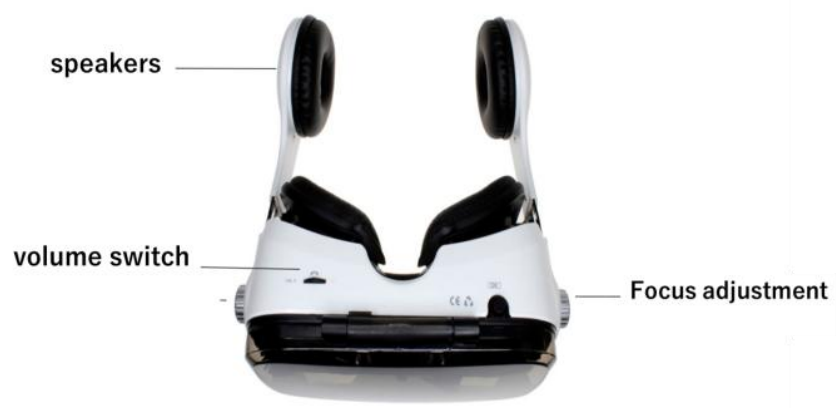

Figure. 1 3D-HMD(head mounted display)

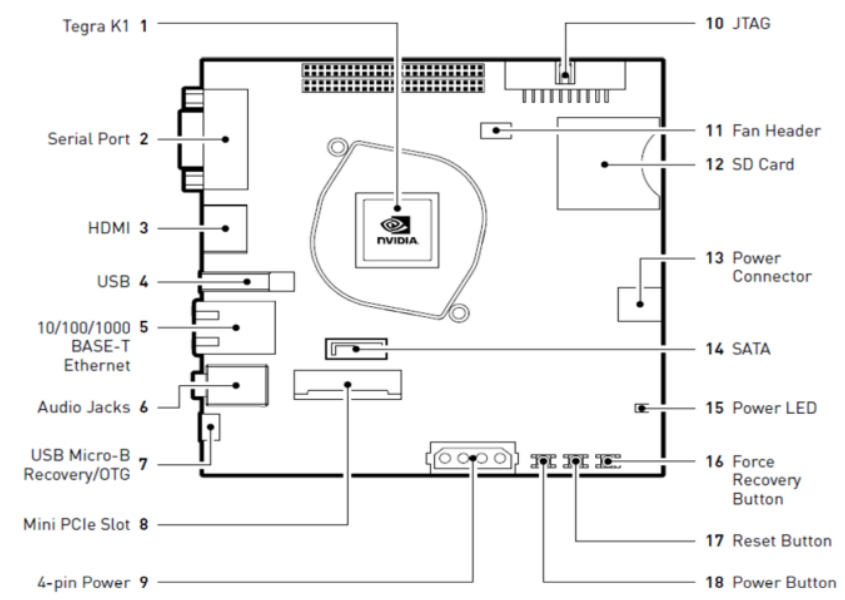

Figure.2 The image processing box

terminal connected by Wi-Fi. The cameras feature two horizontally installed miniature CMOS color image sensors (external dimensions $25 \mathrm{~mm}$ x $25 \mathrm{~mm}$, horizontal angle of view $30-120 x$ ). The display unit has a resolution at least as good as VGA (default resolution $640 \times 480$ ) at both left and right. Adjusting screws are installed at both sides to allow focus adjustment and binocular parallax. The image processing box (Fig. 2) is $13.5 \mathrm{~cm} \mathrm{x} 13.5 \mathrm{~cm} \mathrm{x} 3 \mathrm{~cm}$ and weighs $200 \mathrm{~g}$. It is considerably more compact overall than the image box previously developed by the lead researcher (30 cm x $25 \mathrm{~cm} \times 9 \mathrm{~cm} ; 3 \mathrm{~kg}$ ) and can be worn on the body. Built-in speakers were also added to enable the use of an audio guide. The HMD weighs about $400 \mathrm{~g}$ and could not be reduced to the initial target weight of below $200 \mathrm{~g}$. This was because of the incorporation of speakers, as well as the addition of head cushioning and a belt to enhance the comfort of the study participants at the expense of a lower weight. However, the use of the head protection belt in this HMD eliminated the problem of an overly bent neck position otherwise induced by the weight of the cameras and HMD display. As a result, it was possible to achieve specifications (1) and (3) of the project targets. 
III-1-2. Image processing function modes

In addition to $10 \mathrm{x}$ magnification, the device was given the following four display functions as image processing functions. As a result, it was possible to achieve specification (2) of the Methods. The four functions were standard display, binary (white-on-black) display, reverse video (color inversion) display, and display with a moving alert symbol. than small character size if the magnification of the HMD goes up. This was because the limited central processing unit (CPU) processing speed of the image processing computer used for this HMD system inevitably gave a slightly low image resolution, resulting in a loss of clarity when magnified characters were displayed. This can be quickly improved by changing to a high-speed CPU. Distant objects were assessed as easier to see when using the HMD compared with a

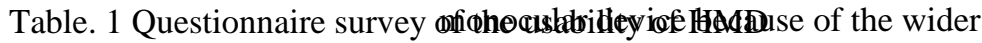

\footnotetext{
Q1 Is it easy to see the sentence (character size is 10.5 point font) by use of $\mathrm{HMD}$ ?

Q2 HMD can do the size of the character is possible up to ten times. Is it enough ?

Q3 Are there dizziness and nausea, etc. when you can read the character and scenery with HMD ?

Q4 Are there dizziness and nausea, etc. when your head and body are moved with HMD ?

Q5 Is it more easy to read the large characters (character size is 16 point fon) with HMD than with a commercialized magnifier reading machine ?

Q6 Is it more easy to use HMD than a commercialized magnifier reading machine ?
}

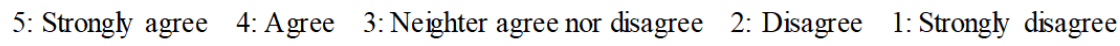

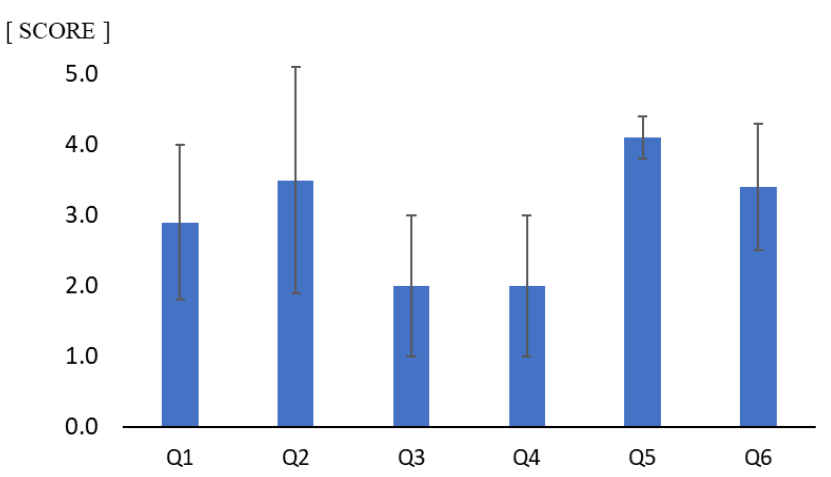

Figure 3. The results of the questionnaire survey of the usability of HMD

\section{III-1-3. External input display mode}

A high-definition image unit compatible with HDMI input was connected by USB so that external images could be used. Images could be displayed on the HMD when connected to a device with an HDMI output terminal (e.g., video camera, PC, whiteboard). As a result, it was possible to achieve specification (4) of the project targets.

\section{III-2. Assessment of specifications by using the HMD}

The Questionnaire survey of the usability of the prototype HMD was conducted in 10 participants ( 9 men, 1 woman; age range, 20-22 years) with visual impairment (low vision). The participants had visual impairment from grade 5 to grade 2, and corrected visual acuity ranging from 0.01 to 0.2 .

After putting on the HMD, reading the material, and looking around the room, the participants assessed ease of reading (ease of seeing) on a 5-point scale (from $1=$ worst assessment to $5=$ best assessment). All subjects answered the six items of the questionnaire survey (Table 1). The results of the questionnaire survey of the usability of HMD showed that the score of the Q5 was the best point (Figure 3). According to the Q1 and Q5, it was found that when the large character size was 16-point font, the mean assessment value among nine participants was high, at 4.1, but with the sentence (character size is 10.5 point font) of the character size, this value was only 2.9. The subject can see the large character size better

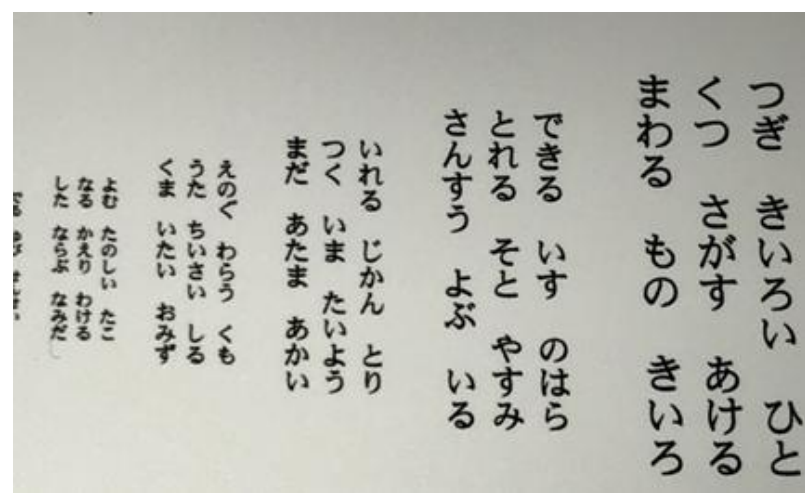

Figure 4. A chart of MNREAD-J

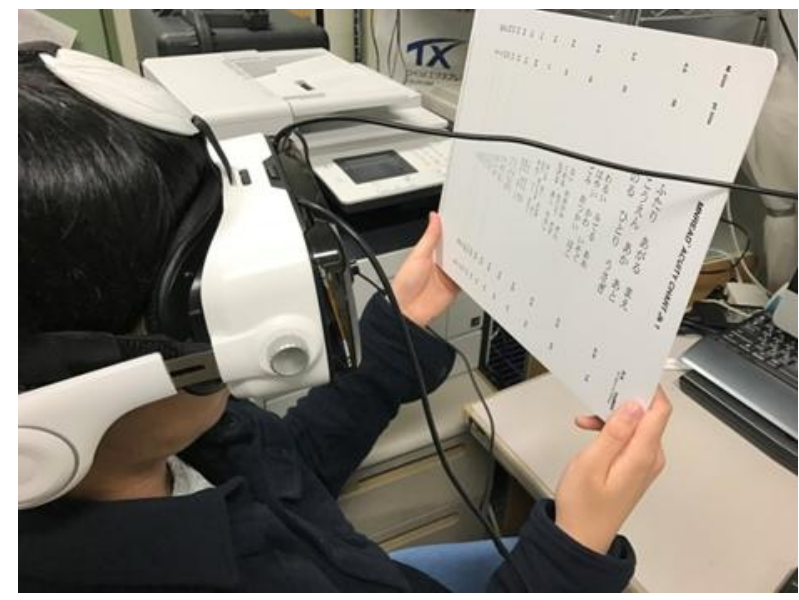

Figure 5. A scene that a subject tried to read a chart of MNREAD-J with new 3D-HMD

visual field provided by the HMD. The participants also felt that the HMD could be useful in helping people with low vision to perform manual tasks, because when performing tasks with a loupe, the distance from the target object cannot be discerned, whereas the 3D capability of the HMD also allows distance to be perceived while the object is being magnified.

The MNREAD-J Acuity test (Fig. 4) was performed both with and without wearing the HMD (Fig. 5), although only 
one participant (male) with low vision was able to perform it. The test required the participant to read continuously from a chart of large point-size characters while reading speed and number of misread characters were measured. When the critical print size calculated from these results was converted to normal visual acuity, the participant's converted visual acuity was 0.066 with the naked eye and 0.132 when wearing the HMD, representing a twofold improvement with the HMD. This test takes at least 1 hour, and only one of the volunteers had sufficiently good visual acuity and concentration to be able to read for that length of time.

\section{CONCLUSIONS}

We developed and tested wearable 3D-HMD with built-in miniature cameras and are capable of performing rapid $3 \mathrm{D}$ magnification of objects captured by the cameras before presenting these images to the display for vision loss and support activities of daily living in older people and those with low vision. The results showed that the 3D-HMD might be effective for the visually handicapped person and the elderly to assist the reduced visual acuity. It was suggested that the improvement in converted visual acuity using the HMD could enhance the accuracy (e.g., precision, speed) of communication aimed at the visually impaired compared with existing devices. In the future, this HMD needs to be studied in a larger sample using several reliable test methods that take less time and thus place less burden on participants. Moreover, we plan to improve the usefulness and applicability of this device by conducting studies in a large sample with versatile visual impairments.

\section{ACKNOWLEDGMENT}

The authors thank the subjects for their participation.

\section{FUNDING}

This work was supported by Northern Advancement Center for Science and Technology and JSPS KAKENHI Grant Number JP 17H02132.

\section{REFERENCES}

[1] Zhao, Y., et al. "Enabling People with Visual Impairments to Navigate Virtual Reality with a Haptic and Auditory Cane Simulation,” Proc. CHI '18, No. 116 (14 pages), 2018.

[2] Miura, T., Ando, G. et al. "Virtual Museum for People with Low Vision: Comparison of the Experience on Flat and Head-Mounted Displays," LNCS 2018;10897:246-249.

[3] Tanaka T, Sugihara S, Nara H, et al.: A preliminary study of clinical assessment of left unilateral spatial neglect using a head mounted display system (HMD) in rehabilitation engineering technology. J Neuroeng Rehabil, 2005, 2: 31.

[4] Tanaka T, Ifukube $T$, Sugihara $S$, et al.: A case study of new assessment and training of unilateral spatial neglect in stroke patients: effect of visual image transformation and visual stimulation by using a Head Mounted Display system (HMD). J Neuroeng Rehabil, 2010, 7: 20 .

[5] Bailey I.L., et al: The design and use of a new near-vision chart. Am. J. Optom. Physiol.Opt., 57: 378-388, 1980.

[6] Faye E.E.: Clinical Low Vision. 45-60, Little Brown, Boston, 1984.

[7] Legge G.E., et al: Psychophysics of reading. II. Low Vision. Vision Res. 25: 253-265,1985.

[8] Rosenthal B.P., et al: Functional Assessment of Low Vision. 1-18, Mosby, St. Louis, 1996.

[9] Legge G.E., et al: Psychophysics of reading. VIII. The Minnesota Low-Vision Reading Test. Optom. and Vis. Sci. 66: 843-853,1989.
[10] Bane M.C., et al: Reading with magnification determined by the MNREAD and Lighthouse Near Acuity Tests in patients with Age-Related Maculopathy (AMD). ARVO Abstracts. Invest. Opthalmol. \& Vis. Sci. 36:S532, 1995.

[11] Bailey J.E., et al: Assessing reading ability in normal and low vision using the MNREAD Reading Acuity Chart: Preliminary results. In V. Lakshminarayanan (Ed), Basic and Clinical Applications of Vision Science, 247-250, Kluwer Academic Publishers, the Netherlands, 1997.

[12] K. Oda, et al: Effect of character size on reading Japanese. ARVO Abstracts. Invest. Opthalmol. \& Vis. Sci. 39: S175, 1998. 